\title{
Common, Rare, and Individual Variations of Mitochondrial DNA Associated with Diseases or Longevity
}

\author{
Masashi TANAKA ${ }^{1,2, *}$ and Kunio YAGI ${ }^{1,2}$ \\ ${ }^{1}$ Gifu International Institute of Biotechnology and ${ }^{2}$ Institute of Applied \\ Biochemistry, Yagi Memorial Park, Mitake, Gifu 505-0116, Japan
}

\begin{abstract}
Summary The human nuclear genome is now being extensively analyzed in search for single nucleotide polymorphisms (SNPs) that are useful for predicting susceptibility to various diseases. To identify SNPs associated with diseases or longevity in the human mitochondrial genome, we have started analyzing the entire sequences of mitochondrial DNA (mtDNA) from 576 individuals, including 96 centenarians, 96 patients with Parkinson's disease, 192 patients with diabetes mellitus with or without severe vascular complications, and 192 young adults with or without obesity. We are going to establish a database for SNPs in the human mitochondrial genome based on these analyses. We assume that the SNPs in the mitochondrial genome are functionally as important as those in the nuclear genome. We hypothesize that the functional property of mitochondria of an individual is genetically influenced by a combination of common, rare, and individual variations in their mtDNA. The construction of human mtDNA database will provide important information for developing an efficient multiplex analysis system for SNPs to predict longevity or susceptibility to age-associated diseases.
\end{abstract}

Key Words: mitochondrial DNA, longevity, Parkinson's disease, centenarian, cytochrome $b$

\section{Functional significance of mitochondrial DNA (mtDNA) polymorphisms}

In spite of the small size of the mitochondrial genome, i.e., 16,569 bp [1, 2], all of the genes (two rRNA genes, 22 tRNA genes, and 13 mRNA genes: $1.5 \times 10^{4}$ $\mathrm{bp}$ ) in the mitochondrial genome are fully expressed in neurons or cardiomyocytes, i.e., the cells that require a large amount of energy to support their activity. Transcripts from the mitochondrial genome account for 25-30\% of the total cellular mRNA. Because certain types of stimulation of cells can influence the expression of the mitochondrial genome, mitochondrial transcripts are frequently detected by differential display. If we assume that 2,000 copies of mtDNA are

${ }^{*}$ To whom correspondence should be addressed. 
present in a cell, the total number of nucleotides functioning in the mitochondrial genome is $3 \times 10^{7}$. This number is only $1 / 200$ of the total number of nucleotides in the nuclear genome $\left(6 \times 10^{9}\right)$. Because only $1.5 \%$ of the nuclear genome is expressed as exons $\left(9 \times 10^{7}\right)$, the effective number of nucleotides in the nuclear DNA is only three times larger than that in mtDNA. If we consider the fact that the evolutionary rate of the mitochondrial genome is 5-10 times higher than that of the nuclear genome, we can conclude that the functional significance of variations within the mitochondrial genome is as profound as that of the variations in the entire nuclear genome. Thus, single nucleotide polymorphisms (SNPs) in the mitochondrial genome might be expected to influence the susceptibility of individuals to various diseases in combination with SNPs in the nuclear genome.

\section{Strategy for elucidating the contribution of human mtDNA variations}

All the nucleotide substitutions in the mitochondrial genome of an individual are tightly linked each other, so that it is difficult to access the functional effect of each substitution. Namely, it is possible that a statistical difference apparently found for one SNP is due to the effect of another SNP in some other region of the genome. Therefore, we have to sequence the entire mitochondrial genome to elucidate the SNP that is directly responsible for the functional difference.

We have tentatively classified mtDNA variations into three groups according to their frequencies: common polymorphisms (frequency $10-50 \%$ ), rare polymorphisms (frequency $1-10 \%)$, and individual variations $(\approx 1 \%)$. The word "common polymorphisms" does not necessarily imply that these polymorphisms are completely normal variants that are neutral in selection. When the SNPs are accompanied by amino acid replacements, it is possible that the functional differences between the common polymorphisms are related to predisposition to longevity or diseases.

To demonstrate the statistical difference in the frequency of a common SNP (at a frequency of $40 \%$ in the control group), we need to analyze approximately 200 individuals for each group. To prove a hypothesis that a rare polymorphism (at $4 \%$ in the control group) contributes to susceptibility to a disease, we need to analyze 2,000 individuals for each group, because only 80 individuals are positive for that SNP. The number of subjects to be examined for analysis of rare SNPs is much larger than that for common SNPs. In the case of individual variations, it is difficult to distinguish whether the particular variation should be regarded as a rare polymorphism that does not significantly alter the function of the gene product or as an individual variation that can be a risk factor predisposing to diseases.

\section{Mitochondrial genotype associated with longevity}

Most centenarians, who have reached at least 100 years of age, are free from life-threatening diseases, such as cardiac infarction, cerebral stroke, diabetes mellitus, or Parkinson's disease. Therefore, centenarians presumably carry genetic 
factors that predispose resistance against these age-related diseases. In line with this notion, we can propose several hypotheses concerning the longevity-associated mitochondrial genotypes. First, a certain mitochondrial genotype may define an efficient mitochondrial respiratory chain that produces less reactive oxygen species. Second, the longevity-associated genotype may encode a mitochondrial oxidative phosphorylation system that is adapted to slow respiration, so that the overall production of ROS is suppressed. Third, the genotype may define a respiratory component that is resistant against attacks by reactive oxygen species. In any case, we suspect that centenarians carry particular genotypes that confer resistance against these age-related diseases. If we can identify such a genotype, we may be able to find the means to prevent age-related diseases and to enjoy long life in good health.

\subsection{Mitochondrial genotype frequent among Japanese centenarians}

To identify possible mitochondrial genotypes associated with longevity, we analyzed the entire coding regions of mtDNA from 11 centenarians [3, 4]. Two nucleotide substitutions, Mt5178A, causing a Leu $\rightarrow$ Met replacement in ND2 (NADH dehydrogenase subunit 2), and Mt8414T, causing a Leu $\rightarrow$ Phe replacement in ATP8 (ATP synthase subunit 8), as well as Mt3010A within the 16S rRNA gene, were more frequently observed in the centenarians than in the controls (Table 1). Among these variations, we focused on Mt5178A, which showed the highest statistical significance of difference, and screened 37 centenarians and 252 healthy blood donors. The frequency of Mt5178A was significantly higher in the centenarians (62\%) than in the blood donors (45\%). To evaluate the effect of mtDNA variations on the occurrence of diseases, we analyzed the frequencies of Mt5178 A and Mt5178C (wild-type) in 338 randomly selected patients. The age distribution of the patients indicated that the frequency of Mt5178C was almost the same as that of Mt5178A among the young patients $($ age $<46)$ but that the

Table 1. Frequencies of nucleotide substitutions in centenarians and controls.

\begin{tabular}{clllrc}
\hline & Nucleotide & \multicolumn{1}{c}{ Gene } & Amino acid & Centenarians & Controls \\
\hline Group I & $8701 \mathrm{~A} \rightarrow \mathrm{G}$ & ATP6 & Thr $\rightarrow$ Ala & $9 / 11$ & $27 / 40$ \\
& $10398 \mathrm{~A} \rightarrow \mathrm{G}$ & ND3 & Thr $\rightarrow$ Ala & $9 / 11$ & $27 / 41$ \\
& $9540 \mathrm{~T} \rightarrow \mathrm{C}$ & CO3 & Leu & $8 / 11$ & $26 / 43$ \\
& $10400 \mathrm{C} \rightarrow \mathrm{T}$ & ND3 & Thr & $10 / 11$ & $25 / 41$ \\
& $10873 \mathrm{~T} \rightarrow \mathrm{C}$ & ND4 & Pro & $9 / 11$ & $25 / 39$ \\
& $12705 \mathrm{C} \rightarrow \mathrm{T}$ & ND5 & Ile & $10 / 11$ & $31 / 41$ \\
& $14783 \mathrm{~T} \rightarrow \mathrm{C}$ & Cytb & Leu & $9 / 11$ & $24 / 39$ \\
& $15043 \mathrm{G} \rightarrow \mathrm{A}$ & Cytb & Gly & $10 / 11$ & $26 / 42$ \\
Group II & $15301 \mathrm{G} \rightarrow \mathrm{A}$ & Cytb & Leu & $10 / 11$ & $26 / 43$ \\
& $5178 \mathrm{C} \rightarrow \mathrm{A}$ & ND2 & Leu $\rightarrow$ Met & $9 / 11$ & $12 / 43$ \\
& $8414 \mathrm{C} \rightarrow \mathrm{T}$ & ATP8 & Leu $\rightarrow$ Phe & $7 / 11$ & $11 / 43$ \\
& $4883 \mathrm{C} \rightarrow \mathrm{T}$ & ND2 & Pro & $9 / 11$ & $12 / 43$ \\
& $14668 \mathrm{C} \rightarrow \mathrm{T}$ & ND6 & Met & $6 / 11$ & $10 / 42$ \\
& $3010 \mathrm{G} \rightarrow \mathrm{A}$ & 16S rRNA & - & $7 / 11$ & $11 / 42$ \\
\hline
\end{tabular}

Vol. 28, No. 3, 2000 
frequency of Mt5178C increased more markedly than that of Mt5178A among the old patients (age $\geq 46$ ). The ratio of $\mathrm{Mt} 5178 \mathrm{~A} / \mathrm{C}$ was significantly lower in the old patients $(86: 166)$ than in both the centenarians $(23: 14)$ and the healthy controls (114:138). Assuming that the old patients would be more prone to age-related diseases, our findings support the concept that to carry a mitochondrial genotype predisposing resistance to adult-onset diseases is one of the genetic factors involved in longevity.

\subsection{Phylogenetic origin of longevity-related Mt5178A}

Reanalysis of the entire mtDNA sequences of 43 individuals [5] revealed that the Japanese people can be classified into three genotypes: Mtype A with Mt10398G + Mt5178A, Mtype B with Mt10398G + Mt5178C, and Mtype C with Mt10398A + Mt5178C. Mtype C cannot be distinguished from the mtDNA sequence of Caucasian subjects [1] by the nucleotide substitutions at the 14 sites shown in Table 1. Mtype B differs from Mtype C by the first group of nucleotide substitutions (two nonsynonymous and seven synonymous substitutions). Individuals with Mtype A carry the second group of substitutions (two nonsynonymous and two synonymous substitutions and one substitution in the 16S rRNA gene) in addition to the first group of substitutions. Horai et al. reported that the divergence between Africans and Europeans occurred $143 \pm 18$ thousand years before the present and that the divergence between Europeans and Japanese occurred $70 \pm 13$ thousand years ago [6]. Phylogenetic analysis suggested that both Mtype $\mathrm{C}$ and Mtype A diverged from Mtype B independently several ten thousand years ago. The phylogenetic relationship among these mitochondrial genotypes is shown in Fig. 1. We analyzed mtDNA from 152 German individuals, but none of them carried Mtype A, $14.5 \%$ of them carried Mtype B, and $85.5 \%$ carried Mtype C. When we compared the frequencies of mitochondrial genotypes in Japan, Korea, China, and Germany, Mtype A was most frequent in Japan; Mtype B, in southern China; and Mtype C, in Germany [7]. These observations suggest that the longevity-associated mitochondrial genotype Mtype A found in Japanese centenarians is related to the longest life span known in the world.

\section{Phylogenetic Tree}

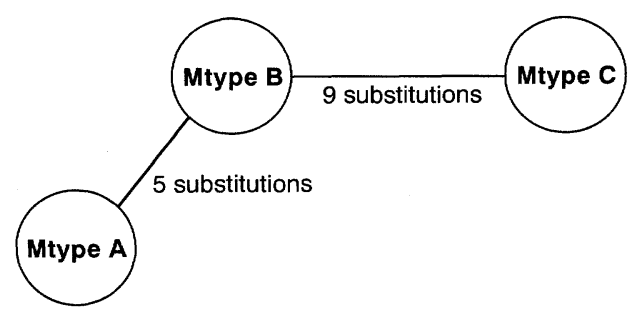

Fig. 1. Phylogenetic relationship among three mitochondrial genotypes according to the common SNPs found in the Japanese population. 


\subsection{Inhibitory effect of Mt5178A on mitochondrial mutagenesis}

Various mutations of mtDNA have been reported in patients with mitochondrial encephalomyopathies and cardiomyopathies. Most of these mutations are present in a heteroplasmic state. The coexistence of both mutant and wild-type mtDNA in patients' tissues suggests that these mutations are new mutations. We examined the frequencies of Mt5178A and Mt5178C among 145 patients with various mitochondrial diseases caused by distinct mitochondrial mutations, and found that the ratio of Mt5178A to Mt5178C (42:103) was significantly lower $(p=0.006)$ than that in the normal controls $(277: 356)$. All 18 patients with Leigh's disease, carrying the $\mathrm{Mt} 8993 \mathrm{~T} \rightarrow \mathrm{G}$ mutation causing a Leu $\rightarrow$ Arg replacement in ATP6, also carried Mt5178C (Tanaka et al., in preparation). These findings suggest that the Mt5178A genotype suppresses the occurrence of mtDNA mutations, especially the Mt8993T $\rightarrow \mathrm{G}$ transversion. Because $\mathrm{T} \rightarrow \mathrm{G}$ transversion can be induced by oxidative damage to DNA, the difference in the occurrence of this mutation may be relevant to the functional differences in mitochondria between Mt5178 A and Mt5178C. We can also speculate that the rate of age-associated accumulation of mitochondrial mutations in somatic cells is different between these mitochondrial genotypes.

\subsection{Anti-atherosclerotic effect of the longevity-associated mitochondrial geno- type}

We evaluated the relationship between the mitochondrial genotypes and atherosclerosis in patients with type 2 diabetes. The intimal plus medial thickness (IMT) of the carotid artery in the diabetic subjects with Mt5178A was significantly less than that in those with Mt5178C. We also observed that the frequency of atherosclerotic plaque in the diabetic subjects with Mt5178A was significantly lower than that in those with Mt5178C. The anti-atherogenic effect of this genotype was estimated to cause an 8-9 years' deceleration of aging-induced atherosclerosis. Thus, the mitochondrial genotype associated with longevity has an anti-atherogenic effect, at least in diabetics [8]. We also evaluated the relationship between mitochondrial genotypes and the complications in patients with type 1 diabetes whose duration of the disease was longer than ten years. Although there was no significant difference in the frequency of retinopathy between the patients with Mt5178A and those with Mt5178C, the frequency of nephropathy was significantly lower in the patients with Mt5178A than in those with Mt5178C [9]. These results indicate that, although the mitochondrial genotypes are not directly linked to the occurrence of diabetes, they would influence the complications of diabetes.

\section{Approaches to rare SNPS in $m t D N A$}

Now we discuss the rare SNPs recently identified in patients with Parkinson's disease. Table 2 shows the rare SNPs identified in the cytochrome $b$ gene among patients with Parkinson's disease or centenarians. The frequencies of Ala193Thr and Gly251Ser were significantly higher among patients with Parkinson's disease 
than among centenarians. The Ile372Val replacement was also more frequent among patients with Parkinson's disease than among centenarian, but this difference was not statistically significant. In contrast, the frequency of Asn260Asp was lower among patients with Parkinson's disease than among centenarians, although the difference in the frequencies was again not significant. Table 3 compares the amino acid sequences surrounding the Gly251Ser replacement site in the cytochrome $b$ subunit of various mammalian species. Because this region is phylogenetically highly conserved, the $\mathrm{Mt} 15497 \mathrm{G} \rightarrow \mathrm{A}$ causing the Gly251Ser replacement is regarded as a radical mutation.

Gly251 is located in the Qo site (the outer ubiquinone binding site) in the vicinity of Glu271, which plays an important role in the quinone binding. When the Gly 251 is replaced by a Ser residue, the Ser 251 forms a hydrogen bond with Glu271. The constraints on the movement of Glu271 by the hydrogen bond with Ser251 would be expected to alter the binding of quinone to the Qo site. It is unknown, however, whether this amino acid replacement actually affects the electron transfer and proton motive functions of the cytochrome $b c_{1}$ complex. These data suggest that to carry certain rare SNPs is a risk factor of Parkinson's disease.

\section{Characteristics of individual mtDNA variations}

One of the difficulties in identifying mtDNA variations predisposing to

Table 2. Comparison of frequencies of rare SNPs in the cytochrome $b$ gene between patients with Parkinson's disease and centenarians.

\begin{tabular}{|c|c|c|c|c|c|c|c|c|}
\hline \multirow{2}{*}{$\begin{array}{c}\text { Nucleotide } \\
\text { change }\end{array}$} & \multirow{2}{*}{$\begin{array}{c}\text { Amino acid } \\
\text { change }\end{array}$} & \multicolumn{3}{|c|}{ Parkinsonian } & \multicolumn{3}{|c|}{ Centenarian } & \multirow{2}{*}{$p$ value } \\
\hline & & Mut & Wild & $\%$ Mut & Mut & Wild & $\%$ Mut & \\
\hline $15323 \mathrm{G} \rightarrow \mathrm{A}$ & Ala193Thr & 6 & 84 & $6.7 \%$ & 0 & 64 & $0.0 \%$ & 0.04 \\
\hline $15497 \mathrm{G} \rightarrow \mathrm{A}$ & Gly251Ser & 6 & 81 & $6.9 \%$ & 0 & 74 & $0.0 \%$ & 0.03 \\
\hline $15524 \mathrm{~A} \rightarrow \mathrm{G}$ & Asn260Asp & 1 & 86 & $1.1 \%$ & 4 & 59 & $6.3 \%$ & 0.16 \\
\hline $15860 \mathrm{~A} \rightarrow \mathrm{G}$ & Ile372Val & 5 & 82 & $5.7 \%$ & 0 & 59 & $0.0 \%$ & 0.08 \\
\hline
\end{tabular}

"Wild" denotes the number of individuals with the wild-type nucleotide at each SNP site; and "Mut," the number of individuals with the mutant nucleotide at each site.

Table 3. Amino acid sequences surrounding the Gly251Ser replacement site in the cytochrome $b$ subunit of various mammalian species.

\begin{tabular}{ll}
\hline Mutant & DLLSDPDN \\
\hline Human & DLLGDPDN \\
Bovine & DLLGDPDN \\
Seal & DLLGDPDN \\
Whale & DLLGDPDN \\
Mouse & DMLGDPDN \\
Rat & DLLGDPDN \\
\hline
\end{tabular}


Table 4. Individual variations in the ATP6 gene found only in one individual among 96 obese subjects.

\begin{tabular}{llcc}
\hline Nucleotide & Amino acid & Difference & Conservation status \\
\hline $8669 \mathrm{G} \rightarrow \mathrm{C}$ & Trp48Ser & 177 & Conserved \\
$8764 \mathrm{G} \rightarrow \mathrm{A}$ & Ala80Thr & 58 & Variable \\
$8894 \mathrm{~A} \rightarrow \mathrm{T}$ & Asn123Ile & 149 & Variable \\
$8950 \mathrm{G} \rightarrow \mathrm{A}$ & Val142Ile & 29 & Variable \\
$9053 \mathrm{G} \rightarrow \mathrm{A}$ & Ser176Asn & 46 & Variable \\
$9182 \mathrm{G} \rightarrow \mathrm{A}$ & Ser219Asn & 46 & Conserved \\
$9197 \mathrm{~A} \rightarrow \mathrm{G}$ & Asp224Gly & 94 & Conserved \\
\hline
\end{tabular}

diseases or longevity is the abundance of sequence variations among individuals. We previously analyzed mtDNA from five patients with Parkinson's disease, and identified several variations that replaced phylogenetically conserved amino acid residues with other residues with different physicochemical properties [10]. However statistical analysis failed to demonstrate a significant difference, because of too low frequencies of the variants in both patients and controls.

Table 4 shows individual mutations in the ATP6 gene identified in only one individual among 96 young adult obese subjects. Although the majority of these mutations cause amino acid replacements between similar residues (e.g., Ala80Thr, Val142Ile, Ser176Asn, and Ser219Asn), some of them resulted in amino acid replacements between residues with different physical and chemical properties (e.g., Trp48Ser, Asn 123Ile, and Asp224Gly). Amino acid residues Ala80, Asn123, Val142, and Ser176 are variable among six mammalian species (human, whale, seal, bovine, rat, and mouse). In contrast, the residues Trp48, Ser219, and Asp224 are conserved among these mammalian species, suggesting that these amino acid residues are functionally important. Because these nucleotide substitutions are apparently homoplasmic, and because none of the subjects carrying these substitutions had any clinical symptoms except obesity, these individual mutations would not cause distinct mitochondrial dysfunction. However we cannot exclude the possibility that these individual mutations causing replacements of phylogenetically conserved amino acid residues into unprecedented ones predispose the subjects to adult-onset diseases.

\section{Interpretation of individual mtDNA variations?}

Population genetics has revealed that the majority of mitochondrial amino acid polymorphisms segregated within a particular species are mildly deleterious [11]. We hypothesize that centenarians are free from deleterious mitochondrial variations, genetically hitting the golden mean. To test this hypothesis, we analyzed amino acid variations in cytochrome $b$ of 64 Japanese centenarians, 96 young adults, and 96 patients with Parkinson's disease, by directly sequencing in each case the entire cytochrome $b$ gene $(1,140 \mathrm{bp})$ in their mitochondrial DNA. Seven different amino acid replacements were found in the centenarian group, whereas 14 and 22 replacements were observed in the young adult group and in the Parkin- 
sonian group, respectively, while the majority of individuals ( 50 centenarians, 75 young adults, and 70 patients with Parkinson's disease) carried no amino acid replacements compared with the revised Cambridge reference sequence. Four replacements were shared by these three groups. In the centenarian group, two variants were detected in one individual, and another variant in two individuals. In the young adult group, ten variants that were not detected in the centenarian group were identified. To quantify the degree of deviation from the common amino acid sequence, we calculated the sum of the predicted physicochemical differences between the standard and the altered amino acid residues for each individual based on the difference values reported by Grantham [12], but we excluded shared replacements from summation. The deviation from the standard sequence in the centenarian group (Grantham value 24 and 21 for each one person and 0 for the other 62 persons) was smaller than that in the young adult group (Grantham value 143, 143, 136, 87, 83, 58, 29, 29, 23, and 15 for each of 10 persons and 0 for the other 86 persons).

Three variants that were not found in the centenarian group were shared by both the Parkinsonian group and the young group. Twelve amino acid variants were observed only in the patients with Parkinson's disease. The deviation from the standard sequence in the Parkinsonian group (Grantham value 143 for four persons; 114, 89, 89, 87, and 78 for each person; 58 for five persons; 46, 29, 29, 27 , 27,23 , and 22 for each person; and 0 for the other 74 persons) was significantly larger than either that in the centenarian group ( $p=0.0037$ by the Mann-Whitney $U$ test) or that in the young adult group ( $p=0.019$ by the Mann-Whitney $U$ test).

We previously reported that the Mt5178A genotype was frequently found in Japanese centenarians [4], whereas Ivanova et al. reported that Mt9055A was associated with French centenarians [13]. Few deviations from the standard amino acid sequence might be common to both Asian and European centenarians with different genotypes. We conclude that occupying the golden mean, at least in cytochrome $b$ sequence, is an important genetic factor in longevity.

\section{Perspective}

There are three implications of the present study. First, the presence of various variations in young adults indicates that these variations scarcely influence either the survival of individuals until maturity or the transmission of the genome to the following generations. Second, the absence of certain variations in centenarians as well as their presence in patients with Parkinson's disease indicate that these variations are not beneficial for long survival but that they predispose to adultonset diseases. Third, a multiplex detection system for a variety of mildly deleterious mitochondrial variations in combination with genetic tests for common longevity-associated genotypes should prove useful both for prediction of longevity and for risk analysis of age-related diseases.

This work was supported in part by grants (to MT) from the programs the Project for 
Database Development from the Japan Science and Technology Corporation, Grants-in-Aid for Scientific Research and the Grants-in-Aid for Priority Areas of the Ministry of Education, Culture, Sports, Science and Technology of Japan and the Grants for Longevity Sciences of the Ministry of Health, Labor, and Welfare of Japan.

\section{REFERENCES}

1. Anderson, S., Bankier, A.T., Barrell, B.G., de Bruijn, M.H.L., Coulson, A.R., Drouin, J., Eperon, I.C., Nierlich, D.P., Roe, B.A., Sanger, F., Schreier, P.H., Smith, A.J.H., Staden, R., and Young, I.G. (1981): Sequence and organization of the human mitochondrial genome. Nature, 290, 457-465.

2. Andrews, R.M., Kubacka, I., Chinnery, P.F., Lightowlers, R.N., Turnbull, D.M., and Howell, N. (1999): Reanalysis and revision of the Cambridge reference sequence for human mitochondrial DNA [letter]. Nat. Genet., 23, 147.

3. Gong, J.-S., Zhang, J., Yoneda, M., Sahashi, K., Miyajima, H., Yamauchi, K., Yagi, K., and Tanaka, M. (1998): Mitochondrial genotype frequent in centenarians predisposes resistance to adult-onset diseases. J. Clin. Biochem. Nutr., 24, 105-111.

4. Tanaka, M., Gong, J.S., Zhang, J., Yoneda, M., and Yagi, K. (1998): Mitochondrial genotype associated with longevity. Lancet, 351, 185-186.

5. Tanaka, M., and Ozawa, T. (1994): Strand asymmetry in human mitochondrial DNA mutations. Genomics, 22, 327-335.

6. Horai, S., Hayasaka, K., Kondo, R., Tsugane, K., and Takahata, N. (1995): Recent African origin of modern humans revealed by complete sequences of hominoid mitochondrial DNAs. Proc. Natl. Acad. Sci. U.S.A., 92, 532-536.

7. Umetsu, K., Tanaka, M., Yuasa, I., Saitou, N., Takeyasu, T., Fuku, N., Naito, E., Ago, K., Nakayashiki, N., Miyoshi, A., Kashimura, S., Watanabe, G., and Osawa, M. (2001): Multiplex amplified product-length polymorphism analysis for rapid detection of human mitochondrial DNA variations. Electrophoresis, 22, 3533-3538.

8. Matsunaga, H., Tanaka, Y., Tanaka, M., Gong, J.-S., Zhang, J., Nomiyama, T., Ogawa, O., Ogihara, T., Yamada, Y., Yagi, K., and Kawamori, R. (2001): Antiatherogenic mitochondrial genotype in patients with type 2 diabetes. Diabetes Care, 24, 500-503.

9. Miura, J., Uchigata, Y., Gong, J.-S., Zhang, J., Iwamoto, Y., Yagi, K., and Tanaka, M. (2000): Mitochondrial genotype Mt5178C is associated with diabetic nephropathy in Japanese type-1 diabetic patients, in Diabetes Mellitus: Recent Advances for the 21st Century, ed. by Shichiri, M., Shinn, S., and Hotta, N., Elsevier Science, Tokyo, pp. 271-274.

10. Ikebe, S., Tanaka, M., and Ozawa, T. (1995): Point mutations of mitochondrial genome in Parkinson's disease. Mol. Brain Res., 28, 281-295.

11. Weinreich, D.M., and Rand, D.M. (2000): Contrasting patterns of nonneutral evolution in proteins encoded in nuclear and mitochondrial genomes. Genetics, 156, 385-399.

12. Grantham, R. (1974): Amino acid difference formula to help explain protein evolution. Science, 185, 862-864.

13. Ivanova, R., Lepage, V., Charron, D., and Schachter, F. (1998): Mitochondrial genotype associated with French Caucasian centenarians. Gerontology, 44, 349. 